\title{
Stone Lattices
}

\author{
Adam Grabowski \\ Institute of Informatics \\ University of Białystok \\ Ciołkowskiego 1M, 15-245 Białystok \\ Poland
}

Summary. The article continues the formalization of the lattice theory (as structures with two binary operations, not in terms of ordering relations). In the paper, the notion of a pseudocomplement in a lattice is formally introduced in Mizar, and based on this we define the notion of the skeleton and the set of dense elements in a pseudocomplemented lattice, giving the meet-decomposition of arbitrary element of a lattice as the infimum of two elements: one belonging to the skeleton, and the other which is dense.

The core of the paper is of course the idea of Stone identity

$$
a^{\star} \sqcup a^{\star \star}=\top,
$$

which is fundamental for us: Stone lattices are those lattices $L$, which are distributive, bounded, and satisfy Stone identity for all elements $a \in L$. Stone algebras were introduced by Grätzer and Schmidt in [18. Of course, the pseudocomplement is unique (if exists), so in a pseudcomplemented lattice we defined $a^{\star}$ as the Mizar functor (unary operation mapping every element to its pseudocomplement). In Section 2 we prove formally a collection of ordinary properties of pseudocomplemented lattices.

All Boolean lattices are Stone, and a natural example of the lattice which is Stone, but not Boolean, is the lattice of all natural divisors of $p^{2}$ for arbitrary prime number $p$ (Section 6). At the end we formalize the notion of the Stone lattice $B^{[2]}$ (of pairs of elements $a, b$ of $B$ such that $a \leqslant b$ ) constructed as a sublattice of $B^{2}$, where $B$ is arbitrary Boolean algebra (and we describe skeleton and the set of dense elements in such lattices). In a natural way, we deal with Cartesian product of pseudocomplemented lattices.

Our formalization was inspired by [17, and is an important step in formalizing Jouni Järvinen Lattice theory for rough sets [19, so it follows rather the latter paper. We deal essentially with Section 4.3, pages 423-426. The description of handling complemented structures in Mizar [6] can be found in [12. The 
current article together with [15] establishes the formal background for algebraic structures which are important for [10, 16] by means of mechanisms of merging theories as described in [11.

MSC: 06D15 06E75 03B35

Keywords: pseudocomplemented lattices; Stone lattices; Boolean lattices; lattice of natural divisors

MML identifier: LATSTONE, version: 8.1.04 5.34.1256

The notation and terminology used in this paper have been introduced in the following articles: [1], 25], [2], [3], 9], 26], 23], 4], [13], 28], 20], [14, 8], [5], [27], and [7.

\section{Preliminaries}

Now we state the proposition:

(1) Let us consider a distributive lattice $L$. Then every sublattice of $L$ is distributive.

Let $L$ be a distributive lattice. One can verify that every sublattice of $L$ is distributive.

Let $L_{1}, L_{2}$ be bounded lattices. One can check that $L_{1} \times L_{2}$ is bounded.

From now on $L$ denotes a lattice and $I, P$ denote non empty closed subset of $L$.

Now we state the propositions:

(2) If $L$ is lower-bounded and $\perp_{L} \in I$, then $\mathbb{L}_{I}^{L}$ is lower-bounded and $\perp_{\mathbb{L}_{I}^{L}}=$ $\perp_{L}$.

Proof: Set $c=\perp_{L}$. Reconsider $c^{\prime}=c$ as an element of $\mathbb{L}_{I}^{L}$. There exists an element $c^{\prime}$ of $\mathbb{L}_{I}^{L}$ such that for every element $a^{\prime}$ of $\mathbb{L}_{I}^{L}, c^{\prime} \sqcap a^{\prime}=c^{\prime}$ and $a^{\prime} \sqcap c^{\prime}=c^{\prime}$ by [3, (68), (73)]. For every element $a^{\prime}$ of $\mathbb{L}_{I}^{L}, c^{\prime} \sqcap a^{\prime}=c^{\prime}$ and $a^{\prime} \sqcap c^{\prime}=c^{\prime}$ by [3, (68), (73)].

(3) If $L$ is upper-bounded and $\top_{L} \in I$, then $\mathbb{L}_{I}^{L}$ is upper-bounded and $\top_{\mathbb{L}_{I}^{L}}=$ $T_{L}$.

Proof: Set $c=T_{L}$. Reconsider $c^{\prime}=c$ as an element of $\mathbb{L}_{I}^{L}$. There exists an element $c^{\prime}$ of $\mathbb{L}_{I}^{L}$ such that for every element $a^{\prime}$ of $\mathbb{L}_{I}^{L}, c^{\prime} \sqcup a^{\prime}=c^{\prime}$ and $a^{\prime} \sqcup c^{\prime}=c^{\prime}$ by [3, (68), (73)]. For every element $a^{\prime}$ of $\mathbb{L}_{I}^{L}, c^{\prime} \sqcup a^{\prime}=c^{\prime}$ and $a^{\prime} \sqcup c^{\prime}=c^{\prime}$ by [3, (68), (73)]. 


\section{Pseudocomplements in Lattices}

Let $L$ be a non empty lattice structure and $a, b$ be elements of $L$. We say that $a$ is a pseudocomplement of $b$ if and only if

(Def. 1) $a \sqcap b=\perp_{L}$ and for every element $x$ of $L$ such that $b \sqcap x=\perp_{L}$ holds $x \sqsubseteq a$.

We say that $L$ is pseudocomplemented if and only if

(Def. 2) for every element $x$ of $L$, there exists an element $y$ of $L$ such that $y$ is a pseudocomplement of $x$.

Now we state the proposition:

(4) Every Boolean lattice is pseudocomplemented.

Let us note that every lattice which is Boolean is also pseudocomplemented and there exists a lattice which is Boolean, pseudocomplemented, and bounded.

Now we state the proposition:

(5) Let us consider a pseudocomplemented, lower-bounded lattice $L$, and elements $a, b, x$ of $L$. If $a$ is a pseudocomplement of $x$ and $b$ is a pseudocomplement of $x$, then $a=b$.

Let $L$ be a non empty lattice structure and $x$ be an element of $L$. Assume $L$ is a pseudocomplemented, lower-bounded lattice. The functor $x^{*}$ yielding an element of $L$ is defined by

(Def. 3) it is a pseudocomplement of $x$.

Now we state the proposition:

(6) Let us consider a pseudocomplemented, lower-bounded lattice $L$, and an element $x$ of $L$. Then $x^{*} \sqcap x=\perp_{L}$.

From now on $L$ denotes a lower-bounded, pseudocomplemented lattice.

Now we state the propositions:

(7) Let us consider an element $a$ of $L$. Then $a \sqsubseteq\left(a^{*}\right)^{*}$.

(8) Let us consider elements $a, b$ of $L$. If $a \sqsubseteq b$, then $b^{*} \sqsubseteq a^{*}$. The theorem is a consequence of $(6)$.

(9) Let us consider an element $a$ of $L$. Then $a^{*}=\left(\left(a^{*}\right)^{*}\right)^{*}$. The theorem is a consequence of (8) and (7).

Let us consider a pseudocomplemented, bounded lattice $L$. Now we state the propositions:

(10) $\left(\perp_{L}\right)^{*}=\top_{L}$.

(11) $\left(\top_{L}\right)^{*}=\perp_{L}$.

(12) Let us consider a Boolean lattice $L$, and an element $x$ of $L$. Then $x^{\mathrm{c}}=x^{*}$. Proof: $x^{*} \sqsubseteq x^{\mathrm{c}}$ by (6), [28, (25)]. $x^{\mathrm{c}} \sqsubseteq x^{*}$ by [28, (20)]. 
(13) Let us consider a pseudocomplemented, bounded lattice $L$, and elements $x, y$ of $L$. Suppose $y$ is a pseudocomplement of $x$. Then $y \in$ the set of pseudo-complements of $x$.

(14) Let us consider a pseudocomplemented, bounded lattice $L$, and an element $x$ of $L$. Then $x^{*} \in$ the set of pseudo-complements of $x$. The theorem is a consequence of (13).

\section{Skeleton of a Pseudocomplemented Lattice}

Let $L$ be a lower-bounded, pseudocomplemented lattice. The functor Skeleton $L$ yielding a subset of $L$ is defined by the term

(Def. 4) the set of all $a^{*}$ where $a$ is an element of $L$.

Now we state the propositions:

(15) Let us consider a lower-bounded, pseudocomplemented lattice $L$. Then Skeleton $L=\left\{a\right.$, where $a$ is an element of $\left.L:\left(a^{*}\right)^{*}=a\right\}$. The theorem is a consequence of (9).

(16) Let us consider a lower-bounded, pseudocomplemented lattice $L$, and an element $x$ of $L$. Then $x \in$ Skeleton $L$ if and only if $\left(x^{*}\right)^{*}=x$. The theorem is a consequence of (9).

Let $L$ be a bounded, pseudocomplemented lattice. Let us note that Skeleton $L$ is non empty.

Now we state the proposition:

(17) Let us consider a pseudocomplemented, distributive, lower-bounded lattice $L$, and elements $a, b$ of $L$. If $a, b \in$ Skeleton $L$, then $a \sqcap b \in$ Skeleton $L$. The theorem is a consequence of (16), (8), and (7).

\section{Stone Identity}

Let $L$ be a non empty lattice structure. We say that $L$ satisfies the Stone identity if and only if

(Def. 5) for every element $x$ of $L, x^{*} \sqcup\left(x^{*}\right)^{*}=\top_{L}$.

Now we state the proposition:

(18) Every Boolean lattice satisfies the Stone identity.

ProOF: $x^{*} \sqcup\left(x^{*}\right)^{*}=\top_{L}$ by (12), [28, (21)].

Let us note that every lattice which is Boolean satisfies also the Stone identity and there exists a lattice which is pseudocomplemented and Boolean and satisfies the Stone identity.

Now we state the proposition: 
(19) Let us consider a pseudocomplemented, distributive, bounded lattice $L$. Then $L$ satisfies the Stone identity if and only if for every elements $a, b$ of $L,(a \sqcap b)^{*}=a^{*} \sqcup b^{*}$. The theorem is a consequence of (6) and (10).

Let $L$ be a lattice. We say that $L$ is Stone if and only if

(Def. 6) $L$ is pseudocomplemented, distributive, and bounded and satisfies the Stone identity.

Let us note that every lattice which is Stone is also pseudocomplemented, distributive, and bounded and satisfies also the Stone identity and every lattice which is pseudocomplemented, distributive, and bounded and satisfies the Stone identity is also Stone.

Now we state the proposition:

(20) Let us consider a pseudocomplemented, distributive, bounded lattice $L$. Then $L$ satisfies the Stone identity if and only if for every elements $a, b$ of $L$ such that $a, b \in$ Skeleton $L$ holds $a \sqcup b \in$ Skeleton $L$. The theorem is a consequence of (19), (16), (8), (9), (6), and (10).

In the sequel $L$ denotes a Stone lattice.

Now we state the proposition:

(21) $\top_{L}, \perp_{L} \in$ Skeleton $L$. The theorem is a consequence of (11) and (10).

Let $L$ be a Stone lattice and $a$ be an element of $L$. We say that $a$ is skeletal if and only if

(Def. 7) $a \in$ Skeleton $L$.

One can verify that $\top_{L}$ is skeletal and $\perp_{L}$ is skeletal and Skeleton $L$ is joinclosed and meet-closed.

Let us observe that the functor Skeleton $L$ yields a closed subset of $L$. The functor SkelLatt $L$ yielding a sublattice of $L$ is defined by the term

(Def. 8) $\mathbb{L}_{\text {Skeleton } L}^{L}$.

Observe that SkelLatt $L$ is distributive.

Now we state the proposition:

(i) $\perp_{L}=\perp_{\text {SkelLatt } L}$, and

(ii) $\top_{L}=\top_{\text {SkelLatt } L}$.

The theorem is a consequence of (21), (2), and (3).

Let $L$ be a Stone lattice. Observe that SkelLatt $L$ is Boolean. 


\section{Dense Elements in Lattices}

Let $L$ be a lower-bounded lattice. The functor DenseElements $L$ yielding a subset of $L$ is defined by the term

(Def. 9) $\quad\left\{a\right.$, where $a$ is an element of $\left.L: a^{*}=\perp_{L}\right\}$.

Now we state the proposition:

(23) $T_{L} \in$ DenseElements $L$. The theorem is a consequence of (11).

Let $L$ be a Stone lattice. Note that DenseElements $L$ is non empty.

Let $a$ be an element of $L$. We say that $a$ is dense if and only if

(Def. 10) $a \in$ DenseElements $L$.

Note that $T_{L}$ is dense.

Now we state the proposition:

(24) Let us consider a Stone lattice $L$, and an element $x$ of $L$.

If $x \in$ DenseElements $L$, then $x^{*}=\perp_{L}$.

Let $L$ be a Stone lattice. Note that DenseElements $L$ is join-closed and meetclosed.

Let us note that the functor DenseElements $L$ yields a closed subset of $L$. The functor DenseLatt $L$ yielding a sublattice of $L$ is defined by the term

(Def. 11) $\mathbb{L}_{\text {DenseElements } L}^{L}$.

Note that DenseLatt $L$ is distributive.

Now we state the proposition:

(25) Let us consider a Stone lattice $L$, and an element $a$ of $L$. Then there exist elements $b, c$ of $L$ such that

(i) $a=b \sqcap c$, and

(ii) $b \in$ Skeleton $L$, and

(iii) $c \in$ DenseElements $L$.

The theorem is a consequence of (7), (6), and (8).

\section{An Example: Lattice of Natural Divisors}

Let us consider a prime number $p$. Now we state the propositions:

(26) The set of positive divisors of $p=\{1, p\}$.

Proof: $\left\{p^{k}\right.$, where $k$ is an element of $\left.\mathbb{N}: k \leqslant 1\right\}=\{1, p\}$ by [22, (4)].

(27) The set of positive divisors of $p \cdot p=\{1, p, p \cdot p\}$.

Proof: $\left\{p^{k}\right.$, where $k$ is an element of $\left.\mathbb{N}: k \leqslant 2\right\}=\{1, p, p \cdot p\}$ by [22, (81), (4)]. 
Let $n$ be a non zero natural number. Let us observe that the lattice of positive divisors of $n$ is finite and there exists a Boolean lattice which is complete.

Let $p$ be a prime number. One can check that the lattice of positive divisors of $p$ is Boolean and the lattice of positive divisors of $p \cdot p$ is pseudocomplemented.

Now we state the proposition:

(28) Let us consider a lattice $L$, a prime number $p$, and an element $x$ of $L$. Suppose $L=$ the lattice of positive divisors of $p \cdot p$ and $x=p$. Then $x^{*}=\perp_{L}$.

ProOF: Reconsider $y_{1}=\perp_{L}$ as an element of $L$. For every element $y$ of $L$ such that $x \sqcap y=\perp_{L}$ holds $y \sqsubseteq y_{1}$ by (27), [14, (64)].

Let $p$ be a prime number. Observe that the lattice of positive divisors of $p \cdot p$ satisfies the Stone identity and the lattice of positive divisors of $p \cdot p$ is non Boolean and Stone and there exists a lattice which is Stone and non Boolean.

\section{Products of Pseudocomplemented Lattices}

From now on $L_{1}, L_{2}$ denote lattices, $p_{1}, q_{1}$ denote elements of $L_{1}$, and $p_{2}$, $q_{2}$ denote elements of $L_{2}$.

Let us assume that $L_{1}$ is a bounded lattice and $L_{2}$ is a bounded lattice. Now we state the propositions:

(29) $p_{1}$ is a pseudocomplement of $q_{1}$ and $p_{2}$ is a pseudocomplement of $q_{2}$ if and only if $\left\langle p_{1}, p_{2}\right\rangle$ is a pseudocomplement of $\left\langle q_{1}, q_{2}\right\rangle$.

PROOF: If $p_{1}$ is a pseudocomplement of $q_{1}$ and $p_{2}$ is a pseudocomplement of $q_{2}$, then $\left\langle p_{1}, p_{2}\right\rangle$ is a pseudocomplement of $\left\langle q_{1}, q_{2}\right\rangle$ by [2, (35), (42), (36)]. For every element $x_{3}$ of $L_{1}$ such that $q_{1} \sqcap x_{3}=\perp_{L_{1}}$ holds $x_{3} \sqsubseteq p_{1}$ by [2, (42), (35), (36)]. For every element $x_{4}$ of $L_{2}$ such that $q_{2} \sqcap x_{4}=\perp_{L_{2}}$ holds $x_{4} \sqsubseteq p_{2}$ by [2, (42), (35), (36)].

(30) $L_{1}$ is pseudocomplemented and $L_{2}$ is pseudocomplemented if and only if $L_{1} \times L_{2}$ is pseudocomplemented. The theorem is a consequence of (29).

Let $L_{1}, L_{2}$ be pseudocomplemented bounded lattices. Let us observe that $L_{1} \times L_{2}$ is pseudocomplemented.

Now we state the proposition:

(31) Suppose $L_{1}$ is a pseudocomplemented bounded lattice and $L_{2}$ is a pseudocomplemented bounded lattice. Then $\left\langle p_{1}, p_{2}\right\rangle^{*}=\left\langle p_{1}{ }^{*}, p_{2}{ }^{*}\right\rangle$. The theorem is a consequence of (29).

In the sequel $L_{1}, L_{2}$ denote non empty lattices.

Now we state the propositions:

(32) If $L_{1}$ is a pseudocomplemented bounded lattice and $L_{2}$ is a pseudocomplemented bounded lattice, then $L_{1} \times L_{2}$ satisfies the Stone identity. 
Proof: Set $L=L_{1} \times L_{2}$. For every element $x$ of $L, x^{*} \sqcup\left(x^{*}\right)^{*}=\top_{L}$ by (31), [2, (43), (35)].

(33) If $L_{1}$ is Stone and $L_{2}$ is Stone, then $L_{1} \times L_{2}$ is Stone.

Let $L_{1}, L_{2}$ be Stone lattices. Let us observe that $L_{1} \times L_{2}$ is Stone.

\section{Special Construction: $B^{[2]}$}

From now on $B$ denotes a Boolean lattice.

Let $B$ be a Boolean lattice. The functor carrier $\left(B^{[2]}\right)$ yielding a subset of $B \times B$ is defined by the term

(Def. 12) $\quad\{\langle a, b\rangle$, where $a, b$ are elements of $B: a \sqsubseteq b\}$.

Let us note that $\operatorname{carrier}\left(B^{[2]}\right)$ is non empty and carrier $\left(B^{[2]}\right)$ is join-closed and meet-closed.

Observe that the functor carrier $\left(B^{[2]}\right)$ yields a non empty closed subset of $B \times B$. The functor $B^{[2]}$ yielding a lattice is defined by the term

(Def. 13) $\mathbb{L}_{\text {carrier }\left(B^{[2]}\right)}^{B \times B}$.

Now we state the propositions:

(34) The carrier of $B^{[2]}=\operatorname{carrier}\left(B^{[2]}\right)$.

(35) $\left\langle\perp_{B}, \perp_{B}\right\rangle \in$ the carrier of $B^{[2]}$. The theorem is a consequence of (34).

(36) $\left\langle\top_{B}, \top_{B}\right\rangle \in$ the carrier of $B^{[2]}$. The theorem is a consequence of (34).

Let $B$ be a Boolean lattice. One can verify that $B^{[2]}$ is lower-bounded and $B^{[2]}$ is upper-bounded.

Now we state the propositions:

(37) $\perp_{B^{[2]}}=\left\langle\perp_{B}, \perp_{B}\right\rangle$. The theorem is a consequence of (2).

(38) $\top_{B^{[2]}}=\left\langle\top_{B}, \top_{B}\right\rangle$. The theorem is a consequence of (3).

Let $B$ be a Boolean lattice. One can check that $B^{[2]}$ is pseudocomplemented. Now we state the proposition:

(39) Let us consider a lattice $L$, elements $x_{1}, x_{2}$ of $B$, and an element $x$ of $L$. Suppose $L=B^{[2]}$ and $x=\left\langle x_{1}, x_{2}\right\rangle$. Then $x^{*}=\left\langle x_{2}{ }^{\mathrm{c}}, x_{2}{ }^{\mathrm{c}}\right\rangle$.

Proof: $x \in \operatorname{carrier}\left(B^{[2]}\right)$. Consider $x_{3}, x_{4}$ being elements of $B$ such that $x=\left\langle x_{3}, x_{4}\right\rangle$ and $x_{3} \sqsubseteq x_{4}$. Reconsider $y=\left\langle x_{2}{ }^{\mathrm{c}}, x_{2}{ }^{\mathrm{c}}\right\rangle$ as an element of $L$. For every element $w$ of $L$ such that $x \sqcap w=\perp_{L}$ holds $w \sqsubseteq y$ by (34), [24, (11)], (37), [2, (35)]. $y$ is a pseudocomplement of $x$.

Let $B$ be a Boolean lattice. One can verify that $B^{[2]}$ satisfies the Stone identity and $B^{[2]}$ is Stone.

Now we state the propositions: 
(40) Skeleton $B^{[2]}=$ the set of all $\langle a, a\rangle$ where $a$ is an element of $B$. Proof: Skeleton $B^{[2]}=$ the set of all $\langle a, a\rangle$ where $a$ is an element of $B$ by $(34),(39),[3,(72)]$.

(41) DenseElements $B^{[2]}=$ the set of all $\left\langle a, \top_{B}\right\rangle$ where $a$ is an element of $B$. Proof: Set $L=B^{[2]}$. DenseElements $L \subseteq$ the set of all $\left\langle a, \top_{B}\right\rangle$ where $a$ is an element of $B$ by (34), (37), (39), [21, (30)]. Consider $a$ being an element of $B$ such that $x=\left\langle a, \top_{B}\right\rangle$. Reconsider $y=x$ as an element of $L$. $y^{*}=\left\langle\left(\top_{B}\right)^{\mathrm{c}},\left(\top_{B}\right)^{\mathrm{c}}\right\rangle$.

\section{REFERENCES}

[1] Grzegorz Bancerek. Cardinal numbers Formalized Mathematics, 1(2):377-382, 1990.

[2] Grzegorz Bancerek. Filters - part II. Quotient lattices modulo filters and direct product of two lattices. Formalızed Mathematıcs, 2(3):433-438, 1991.

[3] Grzegorz Bancerek. Ideals Formalized Mathematics, 5(2):149-156, 1996.

[4] Grzegorz Bancerek. Complete lattices. Formalized Mathematics, 2(5):719-725, 1991.

[5] Grzegorz Bancerek. The ordinal numbers Formalized Mathematics, 1(1):91-96, 1990.

[6] Grzegorz Bancerek, Czesław Byliński, Adam Grabowski, Artur Korniłowicz, Roman Matuszewski, Adam Naumowicz, Karol Pąk, and Josef Urban. Mizar: State-of-the-art and beyond. In Manfred Kerber, Jacques Carette, Cezary Kaliszyk, Florian Rabe, and Volker Sorge, editors, Intelligent Computer Mathematics, volume 9150 of Lecture Notes in Computer Science, pages 261-279. Springer International Publishing, 2015. ISBN 978-3319-20614-1. doi $10.1007 / 978-3-319-20615-8 \_17$.

[7] Czesław Byliński. Some basic properties of sets Formalized Mathematics, 1(1):47-53, 1990.

[8] Marek Chmur. The lattice of natural numbers and the sublattice of it. The set of prime numbers. Formalızed Mathematıcs, 2(4):453-459, 1991.

[9] Agata Darmochwał. Finite sets. Formalized Mathematics, 1(1):165-167, 1990.

[10] Adam Grabowski. On the computer-assisted reasoning about rough sets In B. DuninKęplicz, A. Jankowskı, A. Skowron, and M. Szczuka, editors, Internatıonal Workshop on Monitoring, Security, and Rescue Techniques in Multiagent Systems Location, volume 28 of Advances in Soft Computing, pages 215-226, Berlin, Heidelberg, 2005. Springer-Verlag. doi:10.1007/3-540-32370-8_15.

[11] Adam Grabowski. Efficient rough set theory merging. Fundamenta Informaticae, 135(4): 371-385, 2014. doi 10.3233/FI-2014-1129.

[12] Adam Grabowski. Mechanizing complemented lattices within Mizar system. Journal of Automated Reasoning, 55:211-221, 2015. doi:10.1007/s10817-015-9333-5.

[13] Adam Grabowski. Prime filters and ideals in distributive lattices. Formalized Mathematics, 21(3):213-221, 2013. doi 10.2478/forma-2013-0023

[14] Adam Grabowski. On square-free numbers. Formalized Mathematics, 21(2):153-162, 2013. doi $10.2478 /$ forma-2013-0017.

[15] Adam Grabowski. Two axiomatizations of Nelson algebras. Formalized Mathematics, 23 (2):115-125, 2015. doi 10.1515/forma-2015-0012.

[16] Adam Grabowski and Magdalena Jastrzębska. Rough set theory from a math-assistant perspective. In Rough Sets and Intelligent Systems Paradigms, International Conference, RSEISP 2007. Warsaw, Poland, June 28-30, 2007, Proceedings, pages 152-161, 2007. doi:10.1007/978-3-540-73451-2_17.

[17] George Grätzer. Lattice Theory: Foundation. Birkhäuser, 2011.

[18] George Grätzer and E.T. Schmidt. On a problem of M.H. Stone. Acta Mathematica Academiae Scientarum Hungaricae, (8):455-460, 1957.

[19] Jouni Järvinen. Lattice theory for rough sets. Transactions of Rough Sets, VI, Lecture Notes in Computer Science, 4374:400-498, 2007. 
[20] Magdalena Jastrzębska and Adam Grabowski. On the properties of the Möbius function. Formalized Mathematics, 14(1):29-36, 2006. doi 10.2478/v10037-006-0005-0.

[21] Jolanta Kamieńska and Jarosław Stanisław Walijewski. Homomorphisms of lattices, finite join and finite meet. Formalized Mathematics, 4(1):35-40, 1993.

[22] Rafał Kwiatek. Factorial and Newton coefficients. Formalized Mathematics, 1(5):887-890, 1990.

[23] Rafał Kwiatek and Grzegorz Zwara. The divisibility of integers and integer relatively primes. Formalized Mathematics, 1(5):82y-832, 1990.

[24] Robert Milewski. More on the lattice of many sorted equivalence relations Formalized Mathematics, 5(4):565-569, 1996.

[25] Andrzej Trybulec. Enumerated sets. Formalized Mathematics, 1(1):25-34, 1990.

[26] Michał J. Trybulec. Integers Formalized Mathematics, 1(3):501-505, 1990.

[27] Zinaida Trybulec. Properties of subsets Formalized Mathematics, 1(1):67-71, 1990.

[28] Stanisław Żukowski. Introduction to lattice theory. Formalized Mathematics, 1(1):215222, 1990.

Received October 22, 2015 